\title{
Lifeguard for Robotic Surgery Assistance "LIGRA": An Interactive Platform centralizing Information and Control in Robotic Surgery
}

\author{
Jean Vaucher, Hannes Bleuler (Member, IEEE)
}

\begin{abstract}
A crying need for standardized safety management in health-care in conventional and in robotic surgery in particular has been identified. The same will, which has led to safer air transportation, can be a great source of inspiration for health-care. This paper proposes an interactive platform for the operating room with robotic surgery in view of an efficient safety implementation.
\end{abstract}

\section{State of The ART}

The international air traffic system operates at an incredibly high level of safety in view of the complexity, the potential for disaster and the number of passengers transported. If this safety record is compared to the one of the health care system of any country, it will bear no comparison, even when only "avoidable" malfunctions are traced. Why is this? A quick glance at the reasons for this crying discrepancy reveals the following facts:

In the medical domain, human errors can lead to potentially lethal accidents. Nevertheless the scope of such errors is often limited to a single victim. On the contrary, the crash of a civil airplane and its subsequent mass of victims has a worldwide emotional impact. It is largely relayed by the medias, and the costs and subsequent needs of identifying responsibilities lead to more spectacular investigations. This leads to the identification of potential technical problems, often organizational, at different levels of the responsibility chain in the entire air traffic sector. Identified problems are then addressed by refinement of the processes, certifications, extensive training etc., and thus eventually mitigating upstream risk. All this demonstrates that awareness of the public and consensus to reduce the risks at a minimum can produce astounding results [1][3].

The effort made to achieve such organizational improvements, their great impact on passenger safety, should be an inspirational source for the medical domain. The present contribution aims at applying this lesson to a small, but rapidly growing segment of the biomedical and health-care system, namely robotic surgery, in view of maximizing safety of the complex environment about to emerge.

*Research supported by FP7 program of the EU

J.V. and H.B. (corresponding author) with Robotics Systems Lab LSRO of EPFL, Lausanne, Switzerland (hannes.bleuler@epfl.ch);
The World Health Organization (WHO) already promotes definition, promulgation, and implementation of checklists and decisional tools among many other similar measures in the domain of surgical robotics.

As an example, WHO provides a simple checklist, which fits on a single sheet, and lists the most important steps to carry before, during and after a surgical operation. According to a pilot study conducted in 2008 , it was shown that morbidity and mortality was reduced by nearly $40 \%$ by introducing such a checklist [2]. First steps in this direction have been taken in the last years, the focus on prevention and proper error management has spread within the European and American medical community (see $[4][5][6][7][8][9])$.

The rapidly increasing number of robotically performed surgeries offers multiple advantages for the patient. Nevertheless it also adds new potential risks, due to the potential technical failures and the misunderstanding of an unexpected situation. It requires that the surgical staff is trained in a new domain. These new risks could be addressed by the information technologies.

This is the state of mind that drove us during our work on the "SAFROS" collaborative project. SAFROS is the acronym for "SAFety in RObotic Surgery" and is a European $7^{\text {th }}$ framework project (to be finished in 2013). The goal of the project is the "development of technologies for patient safety in robotic surgery". Ten partners from public and private organizations participate in this project.

The project implements efficient safety innovations for robotic surgery, such as haptic devices allowing the surgeon to feel interaction forces, and various sensing systems in the operating room, detecting e.g. human / machine potential collisions. EPFL's task within SAFROS is to design and develop a user interface, and provide permanent assistance to the surgical team. The improvement in term of patient safety is assessed by the validation processes defined in the project.

The following describes the motivations which lead to the realization of the "LIfe Guard for Robotic surgery Assistance (LIGRA)". First concrete proposals and algorithms for a solution have been implemented. 


\section{Description of Platform Features}

\section{A. Identifying the needs}

Focusing on the technical gap introduced by the use of the newest technologies, and the fact that surgeons are not trained to maintain and install complex robotic systems, we identify the need of a tool, supporting the surgical staff, allowing them to understand a complex robotic system composed by many parts. Its main function is to assist the team during any regular procedure and especially in case of unexpected events. In order to achieve this, the tool must reflect the structure of the system, identify the composing parts, and offer a high level of usability. LIGRA is intended to make the link between the technical world and the surgical world. An experienced surgical team from Ospedale San Raffaele in Milan, Italy, participated actively in the evaluation of a first version of this tool, through trials, structured interviews and questionnaires. A high level of intuitiveness for the user is among the top priorities.

\section{B. The Dashboard feature}

The main feature of LIGRA is to act as a dashboard, which displays the status of all technical equipment. (Fig. 1) The robotic system used to test the LIGRA solution is the MiroSurge, provided by the German Aerospace Center (DLR) [10]. LIGRA monitors the different controller of the robotic system, and displays the status on a touch screen, where the Graphical User Interface of LIGRA is running.

LIGRA dashboard content is dynamic. We only export an Application Programming Interface (API) for the component that need to report information to the system. It is not realistic to rely on an exhaustive list of components. Therefore, the components register themselves to LIGRA, and all information is displayed in a normalized form on the dashboard, thus having a single representation for the surgical staff. This is important since the idea is to encourage a wide use of LIGRA, and not to master the increasing number of available components, and their multiple potential messages.

Technically, the dashboard is built on the "monitor" message transport middleware, which is part of the MiroSurge solution proposed by DLR. This distributed piece of software runs on every controller node in the system. Using the corresponding client library, controllers can send any message to a central repository, where they are stored into a database. Transport is done through TCP connections, and messages are prioritized by severity. A port of the system has also been developed, in order to interface with the ROS framework from Willow Garage [11]. Any other middleware that provides a reliable message transport system could be used.

LIGRA software itself consists in a web server-client pair, taking advantage of the web browser capabilities to be used as an intuitive and powerful graphical user interface.

An instance of node.js light weighted web server runs and constantly "listens" to any message arriving from the monitor middleware. As soon a new message arrives, the web browser is notified, and the content of the dashboard is updated consequently. The server also answers to some client Representational State Transfer (REST) requests [12], allowing identifying users, and storing their customization preferences, such as the acoustic alerts settings.

In order to reach our goal and to display the different status of monitored items in a standard way, each message sent on the monitor consists in structured data, and this data structure is serialized into the message using the JavaScript Object Notation (JSON) notation [13]. The message

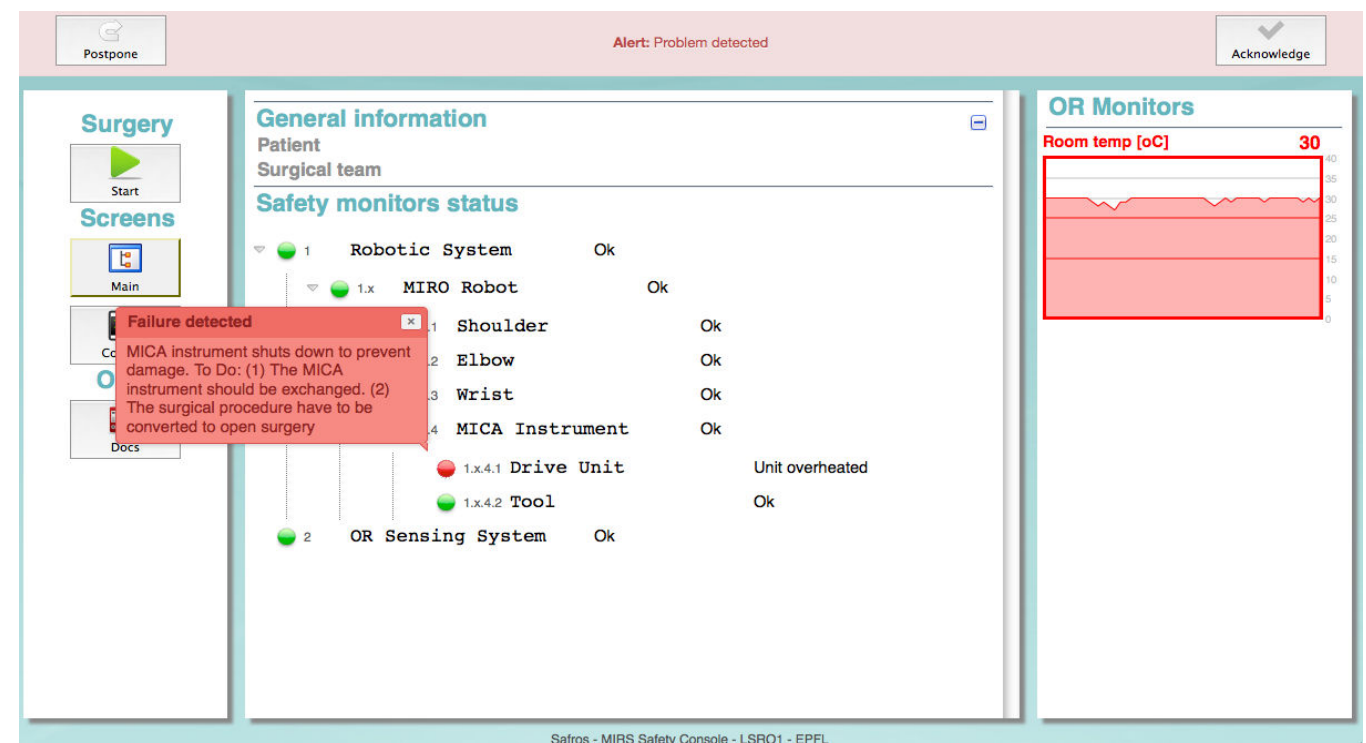

Fig. 1. LIGRA Interface. When a failure occurs an auditory alarm warns the crew. A popup dialog explains the situation and the steps required for recovery. 
contains a unique component identification number, and one or more control commands such as "register" "unregister" or "status update " (Fig. 2).

The component identification numbering scheme is a dot-separated list of integers, thus defining a hierarchy between the components, and representing the belong-to / composed-by relationship. Such hierarchy is represented with a tree graph on the user interface, namely the "safety" tree. All stages can be extended or collapsed in order to view or hide components details. In case a component is reporting a faulty status, it allows the staff to identify which part is faulty on the tree, and therefore what part of the robotic system is not working properly.

The register command is sent at the beginning of the life cycle of the component. Command parameters define the name and the description of the component role. As soon this command arrives, the component is added to the safety tree. The same command can optionally enable a "watchdog" feature. To do so, the component sets a periodic reporting interval. The system then expects to receive a periodic "heartbeat" message at a frequency corresponding to the defined time delay. If the component fails to report its activity in time, it is itself seen and reported as faulty, even if it has not reported any problem explicitly.

The "unregister" command removes the component from the list, and disables the watchdog failure if applicable.

The "status update" command changes the component status displayed on the dashboard. It consists in a textual part which is free and up to the manufacturer, a status severity, and, in case this severity is "warning" or "error" an optional failure code. This code refers to one of the "failures" listed in the central failure repository, where all components documentation is stored. If this failure code is part of the command sent, LIGRA retrieves the documentation related to this code and displays the step-by-step procedure to follow, in order to recover the system. The same documentation repository is also available anytime on LIGRA, allowing the audience to learn about the potential failures in advance and to be, in a way, prepared to react with adequate gestures.

When a command arrives the content of the safety tree is mutated accordingly. Socket.io library is used to push the notification from the server to the web browser, avoiding "polling" and delay. On client side, some javascript code

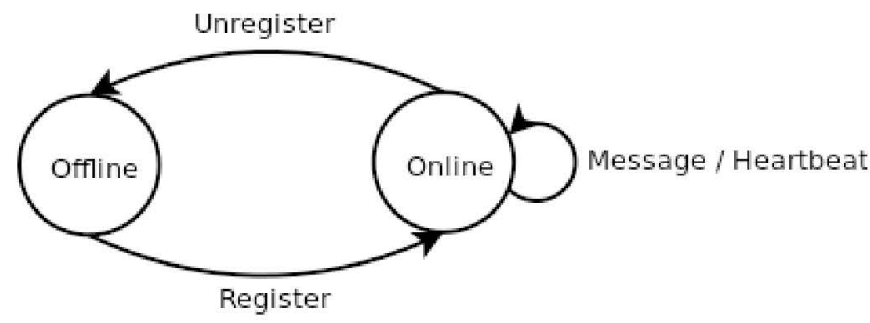

Fig. 2. State machine representation of the component lifecycle and the corresponding LIGRA commands. keeps the domain object model (dom) up-to-date, adding, removing components and updating status texts.

\section{The recording feature}

All the data incoming from the different components connected to LIGRA is logged into a database. At the beginning of a surgical procedure, the surgical staff is authenticated. Their names, their roles, the surgical procedure name are linked to the collected data. The patient data could also be collected, as soon as LIGRA is integrated with the in-house hospital data system. In order to ease this process, it is highly desirable that such systems are standardized at an international level.

This information allows to tag the surgical process, and to retrieve all data concerning a previous intervention from the database. We can see this possibility as a first step in the direction of a "black box" concept.

As it was the case in the air traffic, this recording feature can play an important role in the improvement of patient safety. In case of a problem, a post analysis is then possible and will allow identifying the chain of events and safety-critical items.

\section{Checklists integration}

The impressive impact of the checklist, as discussed above, motivates us to bring this aspect into the LIGRA system. As explained before, WHO provides a checklist which consists in a static list of questions. This list is generic and relevant to any kind of surgical intervention.

Our current integration allows the surgical staff to review all points of this checklist at different point of the surgical process. The items are checked, and the result is stored into the $\log$ of the running surgical process.

In order to keep the checklist simple and thus to facilitate its adoption by the surgical world, the current version remains very concise, addressing only the most important points. By

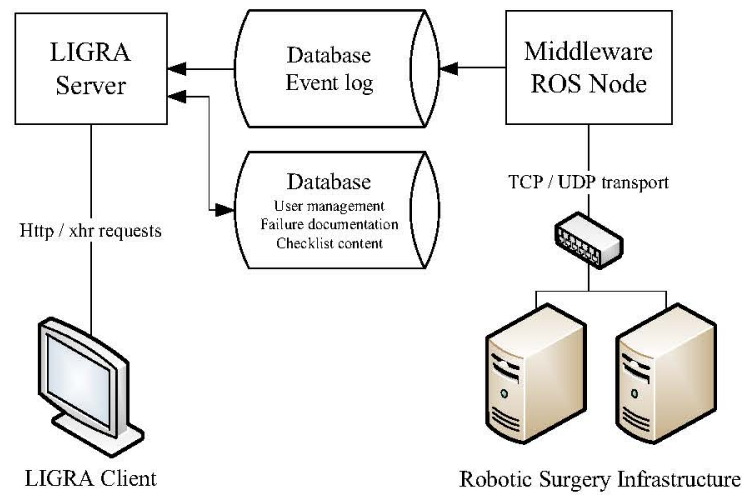

Fig. 3. Layout and integration of the LIGRA system. 
taking advantage of an interactive user interface and through a future the link with e-health system, this concept has strong potential for development in multiple directions:

First, the checklist can vary regarding the current operation, patient specific physiology and take his/her posology in account. Then, the content itself of the checklist will be dynamic, i.e. questions will be displayed conditionally in function of context and of previous answers. This has yet to be implemented.

The complete layout of the LIGRA system is depicted in Fig. 3

\section{RESULTS AND CONCLUSIONS}

The Life Guard for Robotic Surgery Assistance "LIGRA" has been presented to the surgical staff in San Raffaele Hospital. Eight surgeons and one scrub nurse interacted with the system during a simulation of a typical robotic surgery environment. During the session, the different types of failure messages were triggered and presented to the audience.

After this session, users were asked to fill a survey about system usability and how they perceived the enhancement of patient safety. They gave rates on a 5 points Likert scale to different aspects of the user interface. The usability survey presented questions about the structural aspect of the information displayed, and the understandability of its terminology for non-technicians. The survey also contained question about the invasiveness of such a tool in an operating room: we asked to evaluate if the acoustic alerts and the amount of interaction required was adapted in the context of a surgery in progress. The expert robotic surgeons gave an overall mark of 3.75 on 5 to the usability aspect. Regarding the enhancement of patient safety, questions concerned the type of failure cases that the system reports, the approach for presenting an event and the recovery steps. This second questionnaire obtained an overall mark of 4 over 5. This evaluation allows us to collect important additional data and ideas from the expert surgeons on potential improvements. This feedback as well as the evaluation session will be detailed in the presentation.

The work reported here is only a beginning. There is still a lot of development required, and the acceptance of such tools is a long-term process. Nevertheless, we deem interesting to present the work in its current state, with the purpose of stimulating the debate and similar innovations. We believe that it will be only a matter of time before a much more stringent safety management in healthcare will be implemented step by step. Our contribution aims at highlighting at all that could be done in the operating room. Especially at the present time, with rapid introduction of tele-operated surgery (also called robotic surgery), the moment seems appropriate to introduce such procedures. If its usage becomes generalized, it will be an excellent way to introduce and promote new technologies and procedures such as interactive surgical checklists, interlocking decision branches, context dependent individualized operations and greatly enhanced patient safety and comfort. The link with the rapidly expanding e-health technologies is obvious.

This activity should focus on providing innovative content for improve patient safety. Once commonly accepted in the operating room, such procedures will become indispensable not only for immediate malfunction avoidance, but also for traceability, reporting, certification etc., exactly as has happened with the air traffic system and its amazing safety record.

\section{ACKNOWLEDGMENTS}

The authors thank the surgeons of the Ospedale di San Raffaele, Milan, Italy, namely Elettra Oleari, Monica Verga, Angelica Morandi, Alberto Sanna

The authors gratefully acknowledge financing by European FP7 project SAFROS

\section{REFERENCES}

[1] Helmereich R. L, "On error management: lessons from aviation". $\operatorname{BMJ}(2000) ; 320: 781-5$

[2] Haynes AB, Weiser TG, Berry WR, Lipsitz SR, Breizat AH, Dellinger EP, Herbosa T, Joseph S, Kibatala PL, Lapitan MC, Merry AF, Moorthy K, Reznick RK, Taylor B, Gawande AA; "A surgical safety checklist to reduce morbidity and mortality in a global population".

Safe Surgery Saves Lives Study Group. N Engl J Med. 2009 Jan 29;360(5):491-9. Epub 2009 Jan 14

[3] Stahel P. F, "learning from aviation safety: a call for formal readbacks in surgery". Patient Safety in Surgery 2008, 2:21 doi:10.1186/1754-9493-2-21

[4] Makary MA, Mukherjee A, Sexton JB, Syin D, Goodrich E, Hartmann E, Rowen L, Behrens DC, Marohn M, Pronovost PJ: "Operating room briefings and wrong site surgery". J Am Coll Surg 2007, 204:236-43

[5] Michaels RK, Makary MA, Dahab Y, Frassica FJ, Heitmiller E, Rowen LC, Crotreau R, Brem H, Pronovost PJ: Achieving the National Quality Forum's "never events": prevention of wrong site, wrong procedure, and wrong patient operations. Ann Surg 2007, 245:526-32

[6] M. Edington (ed.). The Institute of Medicine Report, "Crossing the Quality Chasm: A New Health System for the 21st Century". Washington, DC: National Academy Press, 2001.

[7] Quality Interagency Coordination Task Force. "Doing what counts for patient safety: Federal actions to reduce medical errors and their impact". Washington, DC: Agency for Healthcare Research and Quality, 2000.

[8] http://www.who.int/patientsafety/en/

[9] Martin A. Makary, J. Bryan Sexton, Julie A. Freischlag,. Anne Millman, David Pryor, Christine Holzmueller, and Peter J. Pronovost, "Patient safety in surgery". Annals of Surgery Volume 243, Number 5 , May 2006

[10] http://www.dlr.de/rm/en/desktopdefault.aspx/tabid-3835/6288 read-9 $047 /$

[11] http://www.willowgarage.com/pages/software/ros-platform

[12] http://en.wikipedia.org/wiki/Representational_state_transfer

[13] http://www.json.org/ 